\title{
Analysis of EXO70C2 expression revealed its specific association with late stages of pollen development
}

\begin{abstract}
Exocyst is an octameric protein complex that mediates the tethering of secretory vesicles at the plasma membrane for exocytosis. In this study, a $1136 \mathrm{bp}$ promoter fragment of exocyst subunit exo70 family protein C2 (EXO70C2) was fused with the b-glucuronidase (GUS) reporter gene and introduced into Arabidopsis thaliana via Agrobacterium tumefaciens. Detail histochemical analysis of EXO70C2-GUS expression in T3 transgenic A. thaliana lines revealed a strong GUS activity at late stages of pollen development. Strong GUS signals were visible from post meiotic pollen, mature pollen and pollen release stages. There was no detectable expression of EXO70C2-GUS during early pollen development and meiosis stages. Consistent with the GUS assay, EXO70C2 transcript profiling using real-time PCR analysis also showed high expression of EXO70C2 at these late stages of pollen development. Further RNA in situ hybridisation revealed the presence of EXO70C2 signals in pollen and as well as anther tapetum. Taken together, these results indicate that, EXO70C2 is specifically expressed in pollen and anther tapetum during the late stages of pollen development, suggesting its function in regulating essential secretory vesicles to support pollen maturation.
\end{abstract}

Keyword: EXO70C2; GUS; Pollen; Promoter; Vesicle secretion 